\title{
Correspondence
}

\section{Chinese Restaurant Syndrome}

SIR,--There is a major criticism that can be raised against the conclusion presented by Morselli and Garattini $^{1}$ that there is no connexion between the ingestion of monosodium glutamate and the Chinese restaurant syndrome. This is that they did not use susceptible subjects. From the very first reports of this condition it was apparent that only a relatively small proportion of normal individuals was susceptible ${ }^{2}$, the proportion being higher among women than among men ${ }^{3}$ (most of the subjects in the experiments reported by Morselli and Garattini were men). Obviously a study of non-susceptible subjects only will not reveal significant reactions.

My own interest in the Chinese restaurant syndrome arises from my suffering from this condition for at least twelve years before the original description of it by Ho Man-Kwok ${ }^{4}$ in 1968. The onset of my recognition of the symptoms occurred when I moved from England to Boston and started to eat in the many excellent Chinese restaurants in that city. Like others ${ }^{3} \mathrm{I}$ was able to prepare a list of "safe" and "to be avoided" Chinese restaurants.

From my personal experiments (unfortunately with only one subject) I can assure the authors that a susceptible subject is capable of recognizing whether monosodium glutamate has been ingested or not: to some extent the amount ingested can also be judged from the severity of the symptoms.

\section{Yours faithfully,}

\section{Jean Himms-HageN}

Department of Biochemistry,

University of Ottawa,

Ottawa 2, Canada.

'Morselli, P. L., and Garattini, S., Nuture, 22\%, 611 (1970).

schaumberg, H. H., and Byck, R., New Engl. J. Med., 279, 105 (1968).

Amos, M., Leavitt, N. R., Marmorek, L., and Wolschina, S. B., New Engl. J. Med., 279, 105 (1968).

- Man-Kwok, Ho, New Engl. J. Med., 278, 796 (1968).

\section{Milk Secretion}

Sir,-Wooding, Peaker and Linzell ${ }^{1}$ seem to portray the knowledge of milk secretion as involving little enlightenment and continuing controversy. In my opinion progress in this field has been impressive and substantial agreement regarding the process is emerging. The two basic milk secretion mechanisms discovered by Bargmann and $\mathrm{Knoop}^{2}$, one involving expulsion of fat globules by envelopment in plasma membrane and the other emptying of Golgi vesicles containing milk proteins through the plasma membrane, are widely accepted as fact. Wooding et al.1 (among others) have reported crescents of cytoplasmic material attached to some (l to 5 per cent) secreted milk fat globules. They combine this observation with their detection of cytoplasmic fragments (concentration ?) in milk as evidence supporting and reviving the theory of apocrine secretion; that is, secretion by cell decapitation with loss of cytoplasm. The unpublished findings of at least three additional groups confirm their observations regarding crescents and all will agree that average milk contains minor amounts of cellular debris.

It seems important to ask how quantitatively significant are these apocrine manifestations. In the case of the cytoplasmic crescents, it can be assumed that average bovine or caprine milk contains about 4 per cent by volume of fat globules. Using the figure by Wooding et al. of 1 to 5 per cent of globules with crescents attached, and assigning an average volume of one-fourth globule volume to the crescents, this aspect of the apocrine mechanism concerns 0.01 to 0.05 per cent of the milk volume. There appears to be little loss of crucial cell machinery into milk such as nuclei, pointed out by Wooding et al..$^{1}$, or mitochondria ${ }^{3}$. In fact the very low levels of phospholipid in milk $(0.02-0.05$ per cent) indi. cate that relatively little cytoplasmic membrane material of any kind is secreted.

\section{Yours faithfully, \\ Stuart Patton}

Division of Food Science and Industry,

The Pennsylvania State University, University Park, Pennsylvania 16802.

1 Wooding, F. B. P., Peaker, M., and Linzell, J. L., Nature, 226, 763 (1970). ${ }^{2}$ Bargmann, W., and Knoop, A., Z. Zellforsch. Mikrosk. Anat., 49, 344 (1959).

${ }^{3}$ Patton, S., Hood, L. F., and Patton, J. S., J. Lipid Res., 10, 260 (1969).

\section{A Definition of Intelligence}

SIR,--In the course of work on cybernetic machines we became increasingly concerned with the problem of intelligence in machines and intelligence in man. An adequate definition of intelligence could not be found in literature and a new one relevant to this problem seemed to be necessary. After due consideration the following definition was formulated:

"Intelligence is that faculty, of mind, by which order is perceived in a situation previously considered disordered."

This definition resolves the potential conflict between so-called intelligent machinery and man since it ascribes, by definition, intelligence only to organisms which have a larger set than a machine can have. Thus machines may have intelligent-like performance but cannot be intelligent because the machine's instruction set is a proper subset of man's instruction set.

Yours faithfully,

H. A. FATMI

R. W. YOUNG

Department of Electronics,

Chelsea College,

University of London,

London SW3.

\section{Phenolase Inhibitors}

SIR,-J. R. L. Walker reported isolation of an unknown phenolase inhibitor from cultures of Penicillium expansum (Nature, 227, 298; 1970). We have found that penicillamine (a degradation product of penicillin) is a potent inhibitor of both mammalian and plant phenolase ${ }^{1}$. It is likely that small amounts of penicillamine accumulating in the culture medium cause the inhibition of $o$-diphenol oxidase.

\section{Yours faithfully,}

\section{K. Prabhakaran}

Research Department,

US Public Health Service Hospital,

Carville, Louisiana 70721. 'Prabhakaran, K.; Harris, E. B., and Kirchheimer, W. F., J. Bact.,
100, 935 (1969). 